\title{
Hybrid low cost flooding scheme for on-demand routing protocols in MANETs
}

\begin{abstract}
Most routing protocols in mobile ad hoc networks (MANETs) use flooding1 to disseminate routing information and to perform route discovery process. As flooding involves querying all network nodes, frequent flooding can rapidly deplete the energy reserved at each node. Therefore, creating competent flooding scheme is a crucial requirement and a Connected Dominating Set (CDS) can be a useful basis of backbone construction in MANETs. However, when the size of CDS becomes too small, certain features in the original network may be lost, as example, the number of broadcasts for a packet to reach its destination can be drastically increased. In this study, a multipoint relay scheme has been investigated and appended as an assistant flooding scheme beside the constructed CDS. The approach used in this concern is the Dominant Pruning (DP) which creates a dynamic dominating set at each broadcasting step. This set is a subset from the neighbor set of the broadcasting node. The incorporation of DP and CDS is made to fill the gaps that may arise within the backbone established by CDS, such that, the number of elongated paths through this backbone is efficiently reduced. Simulation results show that the developed approach outperforms DP and CDS when they are applied individually in terms of the number of flooded control packets, data delivery ratio and average end-to-end delay while keeping the same computation complexity of them.
\end{abstract}

Keyword: Dominant pruning; Dominating set; Flooding cost; Flooding schemes; Mobile ad hoc network 